Acta Crystallographica Section D

Biological Crystallography

ISSN 0907-4449

\section{Cecile Morlot, ${ }^{\mathrm{a}}$ Wieger Hemrika, ${ }^{\text {b }}$ Roland A. Romijn, Piet Gros, ${ }^{\mathrm{c}}$ Stephen Cusack ${ }^{\mathrm{a} *}$ and Andrew A. McCarthy ${ }^{\text {a* }}$}

aEuropean Molecular Biology Laboratory, 6 Rue Jules Horowitz, BP 181, 38042 Grenoble, France, ${ }^{\mathbf{b}} \mathrm{ABC}$ Expression Center, Utrecht University, Padualaan 8, $3584 \mathrm{CH}$ Utrecht, The Netherlands, and ${ }^{\mathrm{c}}$ Department of Crystal and Structural Chemistry, Bijvoet Center for Biomolecular Research, Utrecht University, Padualaan 8, $3584 \mathrm{CH}$ Utrecht,

The Netherlands

Correspondence e-mail: cusack@embl.fr, andrewmc@embl.fr

\title{
Production of Slit2 LRR domains in mammalian cells for structural studies and the structure of human Slit2 domain 3
}

Slit2 and Roundabout 1 (Robo1) provide a key ligandreceptor interaction for the navigation of commissural neurons during the development of the central nervous system. Slit2 is a large multidomain protein containing an unusual domain organization of four tandem leucine-rich repeat (LRR) domains at its $\mathrm{N}$-terminus. These domains are well known to mediate protein-protein interactions; indeed, the Robo1-binding region has been mapped to the concave face of the second LRR domain. It has also been shown that the fourth LRR domain may mediate Slit dimerization and that both the first and second domains can bind heparin. Thus, while roles have been ascribed for three of the LRR domains, there is still no known role for the third domain. Each of the four LRR domains from human Slit2 have now been successfully expressed in milligram quantities using expression in mammalian cells. Here, the crystallization of the second and third LRR domains and the structure of the third LRR domain are presented. This is the first structure of an LRR domain from human Slit2, which has an extra repeat compared with the Drosophila homologue. It is proposed that a highly conserved patch of surface residues on the concave face may mediate any protein-protein interactions involving this LRR domain, a result that will be useful in guiding further studies on Slit2.

\section{Introduction}

Axons are known to travel long distances and follow specific pathways to ultimately reach their correct targets during embryonic development. They are able to achieve this remarkable feat by simplifying the complex pathway into a smaller number of intermediate steps or choice points (Tessier-Lavigne \& Goodman, 1996). One such choice point occurs at the midline, where commissural axons turn longitudinally into specific lateral pathways. They do this by processing a number of different signals emanating from the midline (Garbe \& Bashaw, 2004). Slit and Roundabout (Robo) provide one of the key ligand-receptor interactions that mediate this important choice point (for a recent review, see Dickson \& Gilestro, 2006). Slit and Robo are highly conserved from fruit flies to mammals, but while Drosophila has a single Slit and three Robos, mammals have three Slit proteins (Slit1, Slit2 and Slit3; Brose et al., 1999) and four Robos (Robo1, Robo2, Robo3 or Rig-I and Robo4 or Magic Roundabout; Huminiecki et al., 2002). Slit-Robo signalling is also implicated in vascularization (Carmeliet \& TessierLavigne, 2005) and cancer (Ito et al., 2006; Prasad et al., 2004).

The Slit proteins are large multidomain proteins secreted by the glial cells at the midline (Rothberg et al., 1990). All the slit proteins share a common domain architecture containing four
Received 30 April 2007 Accepted 19 July 2007

PDB Reference: third LRR domain of human Slit2, 2v70, r2v70sf.
(C) 2007 International Union of Crystallography Printed in Denmark - all rights reserved 
leucine-rich repeat (LRR) domains, seven to nine epidermal growth factor-like (EGF-like) domains, a laminin G domain and a cysteine-rich domain at the C-terminus (Brose et al., 1999). Slit2 is cleaved within the EGF-like region by an unknown protease and both fragments have distinct activities (Nguyen Ba-Charvet et al., 2001). The Slit proteins are unusual in containing multiple LRR domains, as LRR domains are usually found in combination with other types of domains (Kobe \& Kajava, 2001). In fact there is only one other protein characterized with multiple LRR domains, the Slitrk family of proteins (Aruga et al., 2003). The only crystal structure to date of a Slit LRR is the third domain from Drosophila (dSlit D3; Howitt et al., 2004). This domain contains five LRRs that are flanked on the $\mathrm{N}$ - and $\mathrm{C}$-termini by cysteine-rich capping domains. This structure also contains some additional residues at the C-terminus that are part of a linker between the third and fourth LRR domains. This linker is covalently linked to the main domain by a disulfide bridge, suggesting that the LRR domains are likely to form a compact arrangement.

LRR domains are well known to mediate protein-protein interactions (Kobe \& Kajava, 2001) and in vitro biochemical studies revealed that the second Slit LRR domain (dSlit D2) is essential for its interaction with Robo (Howitt et al., 2004). These studies also suggest that the fourth LRR domain (dSlit D4) may play an important role in Slit dimerization. Recent studies have shown that the first and second Slit2 LRR domains (Slit2 D1 and Slit2 D2) interact with heparin (Hussain et al., 2006). It is still unclear what, if any, the role of the third LRR domain (Slit2 D3) is, but Slit2 has also been implicated in binding both netrin 1 and laminin 1 (Brose et al., 1999). In order to structurally characterize the Slit-Robo interaction at the molecular level, we initiated a structural project on the Slit2 LRR domains. Here, we present the expression of all four Slit2 LRR domains in a mammalian expression system, the successful crystallization of Slit2 D2 and Slit2 D3 and the structure of Slit2 D3. These results, together with the recent crystallization of the two $\mathrm{N}$-terminal Ig domains of Robo1 (Morlot et al., 2007), will pave the way for a concerted effort in determining the structural basis of the Slit2-Robo1 signalling complex.

\section{Materials and methods}

\subsection{Cloning}

All constructs were produced by PCR amplification using the full-length cDNA coding for human Slit2 (NM_004787) (Piper et al., 2002). Cloning experiments were performed using the following nucleotides ( $5^{\prime}$ to $\left.3^{\prime}\right)$ : S2d1aF, CAG CAC TAG GGA TCC GCG TGC CCG GCG CAG; S2d1bF, CAG CAC TAG GGA TCC CTG AAC AAG GTG GCA CCG CAG; S2d1R, TCG TCG ATC AGC GGC CGC ACT GCA GAC AAA TTC TCG TTT TTG AAC CTC; S2d2F, CAG CAC TAG GGA TCC TTG CAC TGC CCT GCC; S2d2R, TCG TCG ATC AGC GGC CGC TGA ACA ACG GAA TTT CTT GCT TTT; S2d3F, CAG CAC TAG GGA TCC GCT TGC CCT GAA AAG TGT; S2d3R, TCG TCG ATC AGC GGC
CGC ATC ACA AGT GAA GTC CTG AAT; S2d4F, CAG CAC TAG GGA TCC CGC TGT CCT ACT GAA TGT ACT; S2d4R, TCG TCG ATC AGC GGC CGC TTG ACA GGT AAA TTT TTT GGA GGG. The forward primers introduced a BamHI restriction site and the reverse primers introduced a NotI restriction site. The Slit2 PCR products were cloned into three modified pTT3 expression vectors (Durocher et al., 2002) encoding (i) a cystatin signal peptide, (ii) an artificial signal peptide (Barash et al., 2002) and a C-terminal hexahistidine tag or (iii) full-length human growth hormone and a TEV-cleavable hexahistidine tag (Leahy et al., 2000).

\subsection{Cell culture}

Human embryonic kidney cells stably expressing EBNA1 (HEK293-EBNA1) were maintained as suspension growing cells in Freestyle expression medium (Invitrogen) supplemented with $0.2 \%$ FCS and $50 \mu \mathrm{g} \mathrm{ml}^{-1}$ geneticin (Invitrogen). Transient transfections were performed essentially as described by Durocher et al. (2002). Briefly, $24 \mathrm{~h}$ pre-transfection the cells were diluted to $0.3 \times 10^{6} \mathrm{ml}^{-1}$ in Freestyle medium (no additions). DNA-PEI complexes ( $1 \mu \mathrm{g}$ DNA: $2 \mu \mathrm{g}$ PEI per millilitre of suspension culture) were formed in Optimem medium (Invitrogen), immediately vortexed and then added to the culture medium after a 10 min incubation.

\subsection{Small-scale expression tests}

Small-scale transfection tests were performed in parallel to determine if the Slit2 LRR domains were overexpressed and which vector-Slit2 LRR domain combinations give the highest secretion levels. HEK293-EBNA1 cell were suspended in $4 \mathrm{ml}$ cultures on six-well plates and transfected with the vectors containing the individual Slit2 LRR domains. Expression media were sampled $4 \mathrm{~d}$ after transfection and analyzed by SDS-PAGE and Western blotting with an anti-His monoclonal antibody (Novagen).

\subsection{Large-scale expression and purification of Slit2 D3 and Slit2 D4}

11 suspension cultures of HEK293-EBNA1 cells were grown in 31 plastic Erlenmeyer flasks (Corning) and transiently transfected with PEI and the construct giving the highest amount of secreted protein, as determined by the small-scale expression experiments. Expression media containing the various proteins of interest were harvested $5 \mathrm{~d}$ after transfection. Cells were separated from the expression media with a Quixstand Hollow Fibre System (GE Healthcare) using a cartridge with a $0.2 \mu M$ cutoff size. The expression media were subsequently concentrated tenfold and diafiltrated into binding buffer $(300 \mathrm{mM} \mathrm{NaCl}$ and $20 \mathrm{~m} M$ Tris $\mathrm{pH} 8$ ) using the same Quixstand system with a $10 \mathrm{kDa}$ cutoff cartridge. The proteins of interest were purified from concentrated media by batch binding to nickel-Sepharose Fast Flow resin (GE Healthcare) charged with $\mathrm{Ni}^{2+}$. After binding, a column was prepared and washed with binding buffer to remove nonspecifically bound proteins. The Slit2 LRR proteins were then eluted using an imidazole gradient to 
Table 1

Data-collection, phasing and refinement statistics.

Values in parentheses are for the outermost resolution shell.

\begin{tabular}{|c|c|c|}
\hline Protein & Slit2 D2 & Slit2 D3 \\
\hline Space group & $P 2_{1} 2_{1} 2_{1}$ & $P 2_{1}$ \\
\hline $\begin{array}{l}\text { Unit-cell parameters } \\
\left(\AA{ }^{\circ},{ }^{\circ}\right)\end{array}$ & $\begin{array}{c}a=60.4, b=123.5 \\
c=127.7, \alpha=90 \\
\beta=90, \gamma=90\end{array}$ & $\begin{array}{c}a=63.7, b=96.9, \\
c=87.2, \alpha=90, \\
\beta=95.3, \gamma=90\end{array}$ \\
\hline Wavelength (A) & 0.9393 & 0.9393 \\
\hline Resolution $(\AA)$ & $30-2.0(2.1-2.0)$ & $30-3.0(3.2-3.0)$ \\
\hline Completeness (\%) & $94.3(87.1)$ & $92(73)$ \\
\hline$R_{\text {merge }} \dagger(\%)$ & $12.4(36.4)$ & $8.3(42.5)$ \\
\hline$\langle I / \sigma(I)\rangle$ & $10.2(4.6)$ & $12.0(2.6)$ \\
\hline Unique reflections & $61528(7607)$ & $19541(2714)$ \\
\hline Total reflections & $363258(42454)$ & $43534(5968)$ \\
\hline$R_{\text {cryst }} \neq(\%)$ & & 22.2 \\
\hline$R_{\text {free }} \S(\%)$ & & 27.1 \\
\hline \multicolumn{3}{|l|}{ R.m.s. deviations } \\
\hline Bonds $(\AA)$ & & 0.017 \\
\hline Angles $\left({ }^{\circ}\right)$ & & 1.68 \\
\hline
\end{tabular}

$\dagger R_{\text {merge }}=\sum_{h} \sum_{i}\left|I_{h, i}-\langle I\rangle_{h}\right| / \sum\left|\langle I\rangle_{h}\right|$ calculated for the whole data set. $\ddagger R_{\text {cryst }}=$ $\sum|| F_{\mathrm{o}}|-| F_{\mathrm{c}}|| / \sum\left|F_{\mathrm{o}}\right| . \quad \S R_{\text {free }}$ was calculated as for $R_{\text {cryst }}$ using $5 \%$ of the data omitted from the structural refinement.

$250 \mathrm{~m} M$ imidazole. Peak fractions were analysed by SDSPAGE and those fractions containing the relevant Slit2 LRR domain were pooled and dialysed against $250 \mathrm{mM} \mathrm{NaCl}$ and $25 \mathrm{~m} M$ Tris $\mathrm{pH} 8$.

\subsection{Large-scale expression and purification of Slit2 D2}

Slit2 D2 was expressed using a similar protocol to that described above (\$2.4). Slit2 D2 secretion levels were comparable to those of the other domains. Surprisingly, the Slit2 D2 yield was initially very low using the standard protocol described above, which was most probably a consequence of aggregation in the $0.2 \mu M$ hollow-fibre cartridge. The Slit2 D2 expression medium was therefore harvested using centrifugation ( $30 \mathrm{~min}$ at $1000 \mathrm{~g}$ ). The cells were also washed with a high-salt solution to ensure that all the Slit2 D2 was recovered from the cell surface. The concentration and diafiltration were performed using the Quixstand System as described for the other domains. In order to prevent the precipitation of Slit2 D2, as observed in $250 \mathrm{mM} \mathrm{NaCl}$ and $25 \mathrm{~m} M$ Tris $\mathrm{pH}$ 8, dialysis of Slit2 D2 into $500 \mathrm{mM} \mathrm{NaCl}$ and $100 \mathrm{~m} M$ HEPES pH 7 was performed.

\subsection{Crystallization and X-ray data collection of Slit2 D2 and Slit2 D3}

Slit2 D2 and D3 were diafiltered into $10 \mathrm{~m} M$ HEPES pH 7.5 and concentrated to approximately 5 and $7 \mathrm{mg} \mathrm{ml}^{-1}$, respectively, by ultrafiltration. Initial crystallization screens were carried out using a Cartesian crystallization robot at the EMBL Grenoble high-throughput crystallization facility. These initial screens led to diffraction-quality crystals of Slit2 D3. It crystallized as plates after $12 \mathrm{~d}$ from precipitant containing $15 \%$ PEG 4000, $0.2 \mathrm{M}$ ammonium acetate and $0.1 M$ sodium citrate $\mathrm{pH}$ 5.6. The crystals were then flashcooled at $100 \mathrm{~K}$ after transferring them to a solution analogous to the crystallization conditions but containing $35 \%$ PEG
4000. Slit2 D3 crystallized in space group $P 2_{1}$ with four molecules per asymmetric unit and a complete X-ray data set was collected to $3.0 \AA$. The initial crystallization conditions for Slit2 D2 were refined manually using Linbro plates (Hampton Research) and the conventional hanging-drop technique. Slit2 D2 crystallized from precipitant containing 20-24\% PEG 4K, 20\% 2-propanol, $0.1 M$ sodium citrate $\mathrm{pH}$ 5.6. The crystals were then flash-cooled at $100 \mathrm{~K}$ after transferring them through cryogenic conditions containing 40\% PEG 4000 and $0.1 M$ sodium citrate $\mathrm{pH}$ 5.6. A data set to $2.0 \AA$ A resolution was collected from a single Slit2 D2 crystal. An analysis of the systematic absences suggested that the crystal has the symmetry of space group $P 2_{1} 2_{1} 2_{1}$ or the related space group $P 2{ }_{1} 2{ }_{1} 2$ and may contain up to four molecules per asymmetric unit. All X-ray data were collected on beamline ID14-4 at the European Synchrotron Radiation Facility (ESRF) and were integrated and scaled using the XDS suite (Kabsch, 1993). A summary of the data statistics is given in Table 1.

\subsection{Structural solution and refinement of Slit2 D3}

A model of human Slit2 D3 was produced using the previously determined Slit D3 from Drosophila (dSlit D3) as a template (Howitt et al., 2004). In brief, the dSlit D3 LRRs 1-4 were superimposed onto dSlit D3 LRRs 2-5. The N-terminal cap and LRRs 1-4 were then fused with the superimposed LRRs 4-5 and the C-terminal cap to generate a model Slit2 D3 domain containing six LRRs. This search model contained the additional LRR that is present in Slit2 D3 and was successfully used to locate four Slit2 D3 domains with Phaser (McCoy et al., 2005). The crystal structure refinement of Slit2 D3 was performed using REFMAC (Murshudov et al., 1997), with a randomly chosen subset of reflections $(5 \%)$ for calculation of the free $R$ factor. Tight NCS restraints were included throughout the refinement and TLS refinement was used in the final rounds. Model building was carried out with Coot (Emsley \& Cowtan, 2004) and the stereochemical qualities of the protein molecules were validated with PROCHECK (Laskowski et al., 1993). All the crystallographic information is summarized in Table 1 . The figures were prepared with PyMOL (DeLano Scientific).

\section{Results}

The Slit2-domain boundaries were designed using the minimal LRR-domain boundaries predicted by SMART (Letunic et al., 2006). During the initial stages of the project, we tried to produce the Slit 2 constructs in both the cytoplasm and periplasm of Escherichia coli using various expression vectors and E. coli strains. Although the proteins were overexpressed, we were never able to produce any soluble protein. These unsuccessful attempts were followed by expression trials using the baculovirus expression system. Small amounts of soluble protein were detected, but we did not observe any clearly overexpressed protein and also ceased this avenue of protein production. We therefore decided to try a mammalian expression system for the Slit2 LRR domains, as such systems 
are fast becoming routine for the production of secreted proteins (Aricescu et al., 2006).

Slit2 D1 was designed to span residues 27-258. Very low secretion levels were observed in the initial expression trials using cystatin or the artificial signal sequence (Barash et al., 2002) and a C-terminal hexahistidine tag, which might be a consequence of improper folding of this domain. Soluble protein could be detected when secretion was driven by a vector containing full-length human growth hormone. Furthermore, increasing the size of the Slit2 D1 domain by the addition of the N-terminal residues 21-26 also resulted in good secretion with the two signal sequences used, although this production was never scaled up. It is also clear from the Western blot of small-scale productions that Slit2 D1 migrates at a higher molecular weight than predicted (28.4 kDa; Fig. 1$)$. This suggests that Slit2 D1 is glycosylated, probably at the Asn66 and/or Asn186 sites predicted by NetNGlyc (Blom et al., 2004).

Slit2 D2 spans residues 271-479 and was well expressed and secreted. Slit2 D2 runs close to its calculated molecular weight $(24.5 \mathrm{kDa})$ and is therefore unlikely to be glycosylated, as predicted by NetNGlyc (Blom et al., 2004). While the expression of Slit2 D2 was always successful, we had to refine the standard protein-purification protocol used. Both Slit2 (Brose et al., 1999) and Slit2 D2 (Hussain et al., 2006) are known to bind heparin and this is suggested to be of biological significance (Hussain et al., 2006). The cells were therefore washed in high salt to ensure all the expressed Slit2 D2 was recovered from the cell surface. We also discovered that Slit2 $\mathrm{D} 2$ is more soluble and easier to handle at $\mathrm{pH} 7-7.5$, so we reduced the $\mathrm{pH}$ used in the purifications. We recently obtained some diffraction-quality crystals and have collected a complete data set to $2.0 \AA$ resolution. Unfortunately, initial attempts to solve this structure by molecular replacement using the Slit2 D3 domain described below have been unsuccessful and we are currently trying other phasing methods.

Slit2 D3 spans residues 505-713 and was well expressed and secreted. Slit2 D3 runs at a higher molecular weight than calculated $(24.6 \mathrm{kDa})$ and is therefore likely to be glycosylated at the Asn564 and/or Asn623 glycosylation sites predicted by NetNGlyc (Blom et al., 2004). Slit2 D3 was purified using the standard protocol and yielded diffraction-quality crystals from our initial crystallization experiments. These

Figure 2 crystals diffracted well and a complete X-ray data set was collected to $3 \AA$ resolution. The structure was subsequently solved by molecular replacement and has now been refined at $3 \AA$ resolution to a final $R$ factor of $22.2 \%\left(R_{\text {free }}=27.1 \%\right)$. The final model is comprised of four Slit2 D3 molecules in the asymmetric unit and an $\mathrm{N}$-glucosamine carbohydrate covalently linked to Asn623. The models of each of the Slit2 D3 molecules contain 211 of a possible 220 residues. The final models have good geometry (Table 1) and analysis with PROCHECK (Laskowski et al., 1993) showed that $73.7 \%$ of residues were in the most favoured regions, $25.1 \%$ in additionally allowed regions and $1.2 \%$ in generously allowed region of the Ramachandran plot. No residues were located in the disallowed regions.

Slit2 D4 spans residues $726-908$ and has the highest production levels. Slit2 D4 runs as three bands with larger molecular weights than predicted $(21.4 \mathrm{kDa}$; Fig. 1$)$. It is

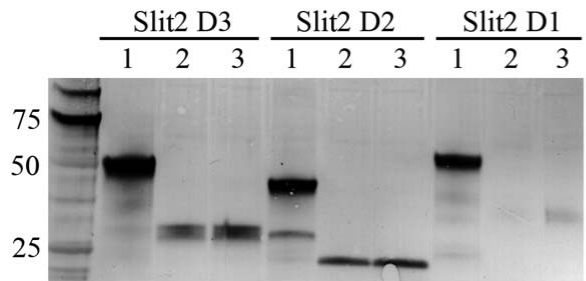

(a)

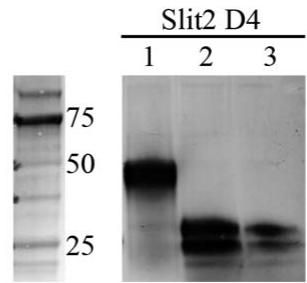

Figure 1

Western blot analysis of secreted Slit2 domains in small-scale transfection experiments. Culture media were collected $4 \mathrm{~d}$ after transfection. $15 \mu \mathrm{l}$ samples were loaded and His-tagged proteins were detected using a monoclonal anti-His antibody. (a) Secretion of the Slit2 domains is driven by (1) human growth hormone, (2) cystatin signal peptide and (3) artificial signal peptide. (b) Secretion of the Slit2 D1 21-258 construct driven by the artificial signal sequence.

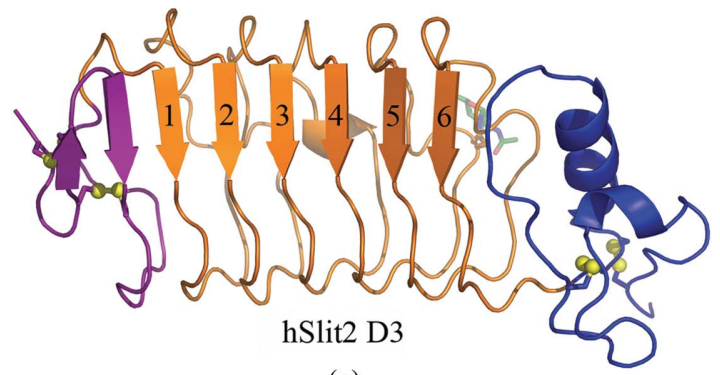

(a)

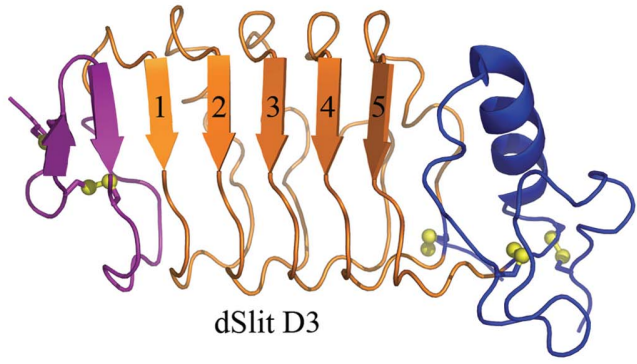

(c)

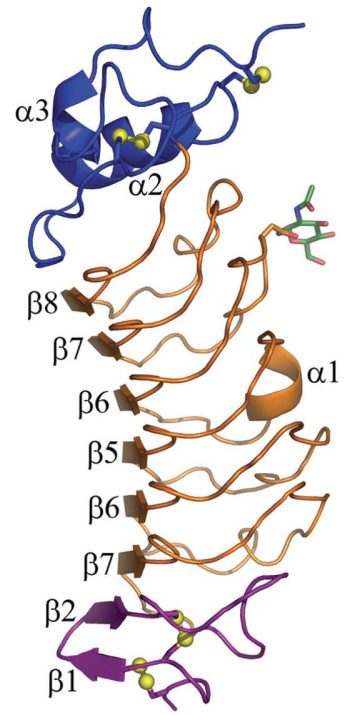

(b)

Structure of Slit2 LRR domains. The N- and C-terminal capping domains are shown in purple and blue, respectively, and the LRR repeats are coloured orange. The disulfide bridges are drawn in yellow ball-and-stick representation and the $N$-acetylglucosamine is drawn in green stick representation. $(a, b)$ Ribbon diagram of human Slit2 D3 in two orthogonal orientations. $(c)$ Ribbon diagram of Drosophila Slit D3. 
therefore likely to be glycosylated at the Asn794 and/or Asn799 glycosylation sites predicted by NetNGlyc (Blom et al., 2004). Slit2 D4 could be purified using the standard protocols and some initial crystallization hits were obtained. Unfortunately, none of these were of sufficient quality for structural analysis. This is probably a consequence of heterogeneous glycosylation on both predicted $\mathrm{N}$-glycosylation sites. We are currently trying mutagenesis and the addition of kifunensine to the culture media followed by EndoH cleavage (Chang et al., 2007$)$ in an effort to improve the crystallization process for further structural studies.

\section{Discussion}

We commenced this work before the publication of the structure of Slit D3 from Drosophila (dSlit D3) and all our constructs lack the additional C-terminal linker region found in this structure (Howitt et al., 2004). This linker is covalently

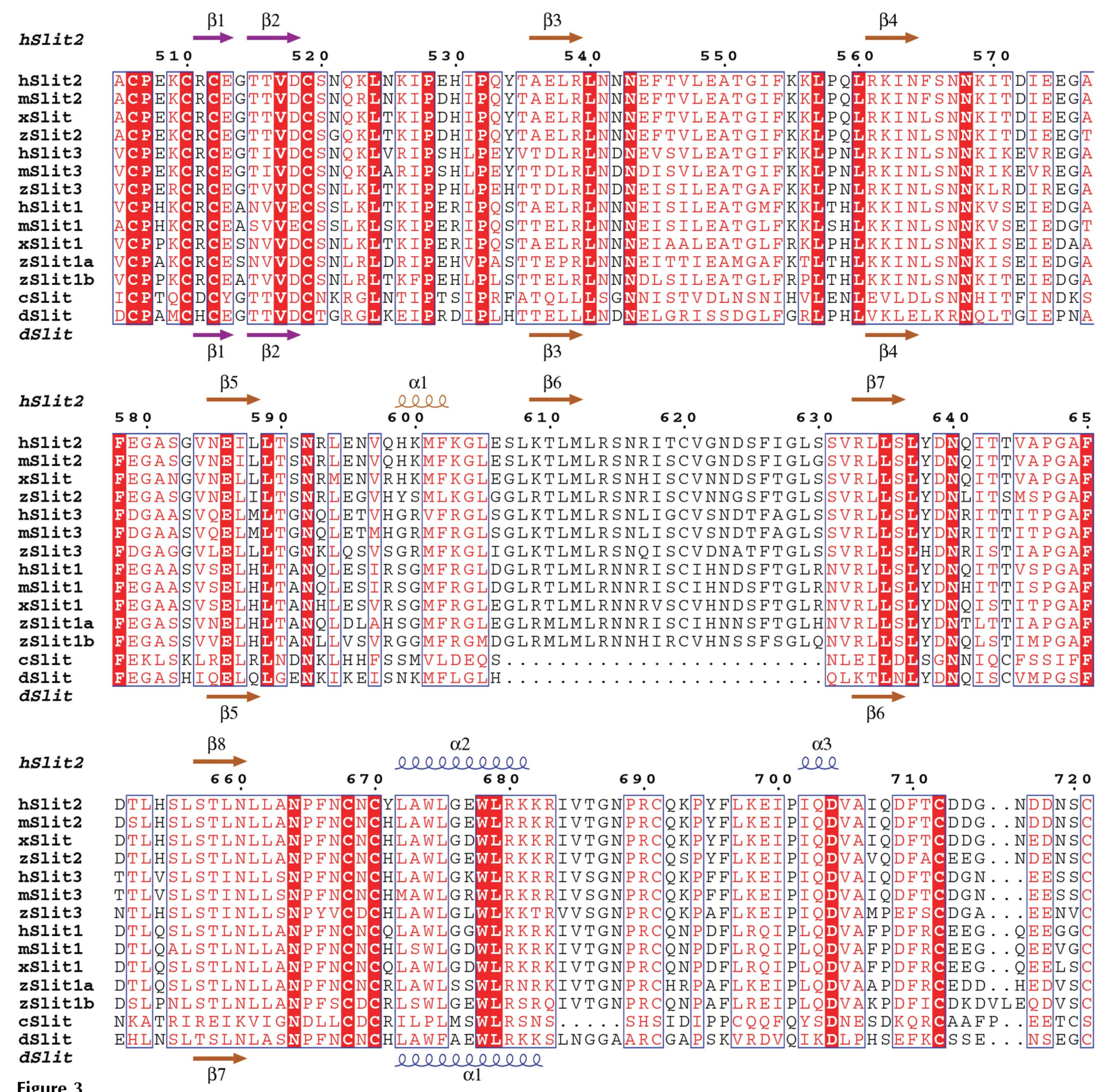

Figure 3

Slit-sequence conservation of vertebrate Slit2 D3. Residue numbering and secondary structures of human Slit2 D3 are indicated on top of the sequences. Sequence alignment of D3 LRR domains: h, human; c, C. elegans; d, Drosophila; m, mouse; x, Xenopus laevis; z, zebrafish. Sequence alignments were performed with ClustalW (Thompson et al., 1994) and ESPript (Gouet et al., 1999). 
attached to the main body of the dSlit D3 domain through a conserved disulfide bond. Our proteins therefore have a free cysteine, but this does not appear to cause any aggregation problems, although Slit2 D2 does aggregate to some extent on concentration. We now have successfully expressed in milligram quantities, three of the four LRR domains from mammalian Slit2. The relatively small amounts of protein and the nanolitre crystallization robot available at EMBL Grenoble have allowed us to crystallize the Slit2 D2 and Slit2 D3 LRR domains. We have now solved the Slit2 D3 structure, the first structure of an LRR domain from human Slit2.

Slit2 D3 is composed of six LRR repeats flanked at the Nand C-termini by cysteine-rich capping domains (Figs. $2 a$ and $2 b$ ). These capping domains are also found in other secreted LRR proteins, such as glycoprotein Ib $\alpha$ (GpIb $\alpha$; Huizinga et al., 2002) and the Nogo receptor (He et al., 2003). Two potential glycosylation sites were identified in the primary structure. However, only one of these, Asn623, was observed to be glycosylated in the structure and is located on the convex face (Fig. 2b). The mammalian Slit2 D3 differs from the recently published dSlit D3 (Howitt et al., 2004) in that it contains an additional LRR (Figs. $2 a$ and $2 c$ ). This extra LRR is present in all vertebrate sequences (Fig. 3) and missing in both Drosophila and Caenorhabditis elegans Slit D3. Despite

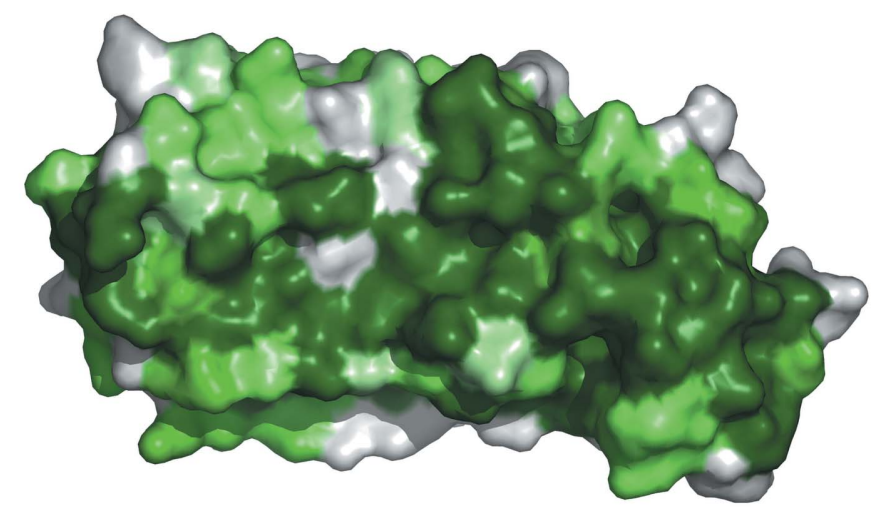

(a)

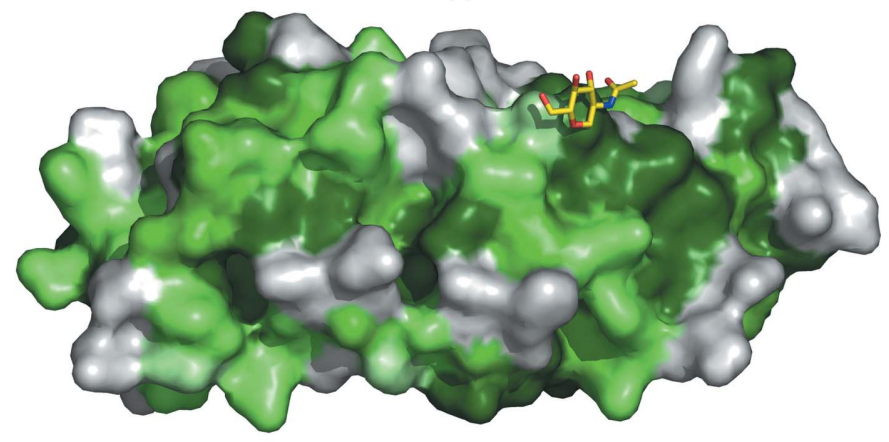

(b)

\section{Figure 4}

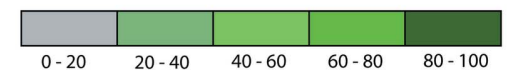

The sequence conservation of surface residues was mapped onto Slit2 D3 and is indicated with a colour gradient from dark green (most conserved) to white (most divergent) in two orientations. (a) Concave face in an orientation identical to Fig. 2. (b) Convex face showing the $N$-acetylglucosamine. The sequence conservation was mapped onto the surface with ConSurf (Landau et al., 2005). the omission of an LRR, dSlit D3 retains a high sequence similarity to Slit2 D3, with $49 \%$ identity over 184 residues (Fig. 3). This is larger than the conservation between Slit2 D2 and D3, where there is only $37 \%$ identity. dSlit D3 is also the closest structural neighbour to Slit2 D3. The N-terminal cap and LRRs 1-3 of Slit2 D3 can be superimposed onto dSlit D3 with an r.m.s.d. of $1.0 \AA$ for $102 \mathrm{C}^{\alpha}$ atoms, with the only major conformational differences occurring in the loops connecting the LRRs. LRRs 5-6 and the C-terminal cap of Slit2 D3 can be superimposed onto LRRs 4-5 and the C-terminal cap of dSlit D3 with an r.m.s.d. of $1.3 \AA$ for $102 \mathrm{C}^{\alpha}$ atoms. Here, the largest conformational difference is observed in the loop following $\alpha 1$, which corresponds to the ' $\beta$-switch' loop of GpIb $\alpha$ (Huizinga et al., 2002).

The Slit2 gene is encoded by 37 exons and demonstrates considerable modularity in the placement of exon-intron boundaries such that LRRs of 24 residues are often encoded by individual exons of 72 bp (Little et al., 2002). In Slit2 D3 the $\mathrm{N}$ - and C-terminal caps are encoded by exons 16 and 20, respectively, LRR 1 is encoded by exon 17, LRRs 2-3 are encoded by exon 18 and LRRs 4-5 are encoded by exon 19 . The last LRR is never predicted bioinformatically in any of the Slit2 LRR domains because it is much smaller and contains only the $\beta$-sheet section, the remainder being incorporated into the C-terminal cap region. A very similar exon organization is seen in both the Slit1 and Slit3 genes. The exon organization of $d$ Slit is different, containing only 19 exons, where the $\mathrm{N}$ - and $\mathrm{C}$-terminal caps are encoded by exons 10 and 13, respectively, LRRs 1-3 are encoded by exon 11 and LRR 4 is encoded by exon 12. Again, the last LRR (LRR 5) is incorporated into the $\mathrm{C}$-terminal cap region. The high degree of remaining sequence similarity and the modularity of the LRR exons make it likely that the human Slit D3 domains may have evolved through the addition of LRR 4 by alternative gene splicing.

Three Slit genes (Slit1, Slit2 and Slit3) are found in most vertebrates and their D3 sequences are highly conserved, with $>66 \%$ identity over $\sim 220$ residues (Fig. 3). The largest sequence conservation occurs on the concave face (Fig. 4). This is not surprising, as LRR proteins are well known to mediate their protein-protein interactions via their concave face (Kobe \& Kajava, 2001). It is therefore plausible that the concave face in Slit2 D3 mediates an interaction between mammalian Slit proteins and another protein, perhaps netrin 1 or laminin 1, both of which are know to interact with Slit2 (Brose et al., 1999). The largest continuous area of surfaceconserved residues occurs between the last three LRRs and the ' $\beta$-switch'. In fact, an identical region of GpIb $\alpha$ mediates one of the two interaction surfaces between GpIb $\alpha$ and the von Willebrand factor A1 domain (Huizinga et al., 2002). Three of the mutants shown to be important for the binding of Drosophila Slit D2 to Robo also map to this region. Interestingly, the most extensive crystal contacts in the structure also map to this location and specifically to residues Met612, Arg614, Ser636, Tyr638, Asn660, Leu662, Val685, Gly687 and Asn688. We therefore propose that these residues will be involved in mediating any interaction involving Slit2 D3. 
The UniProt Slit2 entry (http://www.uniprot.org/entry/ slit2_human) contains three isoforms, two sequence variants and three sequence conflicts. Three of these sequence variations are located in Slit2 D3. The first is a serine-to-proline single-nucleotide polymorphism (SNP) variant at position 636 (Itoh et al., 1998). The second is a sequence conflict, reporting a change from SLKT to KPQN between amino-acid positions 607 and 610, and the last is a L634M conservative mutation (Brose et al., 1999). Interestingly, these differences also map to the concave face and the L634M and S636P mutations map onto or near the conserved surface region identified above. Whether these are of biological significance remains to be determined. No sequence variations were identified in the third domain of the Slit1 UniProt entry and the four SNPs located in the third LRR domain of the Slit3 UniProt entry are either conservative or located on the convex face.

\section{Conclusion}

Transient expression of the four individual LRR domains from Slit2 in mammalian HEK293-EBNA1 cells resulted in the production of sufficient protein for the growth of diffractionquality crystals of the Slit2 D2 and Slit2 D3 domains, and preliminary crystallization hits for the Slit2 D4 domain. The Slit2 D2 domain is known to mediate the interaction between Slit2 and its receptor, Robo1, and we hope to solve the structure of this important domain in the near future and to gain further insights into its molecular interaction with Robo1. The Drosophila Slit protein contains an identical domain architecture to Slit2, with the largest difference occurring in the third domain, where human Slit2 contains an extra LRR (Fig. 2). In addition, only ten of the 23 residues on the concave face of Slit2 D3 available for interaction are conserved in dSlit D3 (Fig. 3). It is therefore probable that these two LRR domains have different binding partners and distinct biological functions in vertebrates and invertebrates. It is also likely that these structural and biological differences may have arisen through alternative gene-splicing events. The largest area of surface-conserved residues in vertebrates occurs on the concave fact of Slit2 D3 and we believe that this face will mediate any interactions with this domain, in particular the last three LRR repeats and the ' $\beta$-switch' loop. We further speculate that Slit2 D3 is the interaction domain for netrin 1, although this will have to be verified experimentally. This hypothesis could account for the silencing effect mediated by a direct Slit2-dependent interaction between Robo1 and the netrin receptor DCC (deleted in colorectal cancer; Stein \& Tessier-Lavigne, 2001). These results may be used in designing further experiments and will help in our understanding of the important Slit-Robo signalling mechanism.

We gratefully acknowledge the use of the EMBL Grenoble high-throughput crystallization facility and thank the EMBL Grenoble/ESRF Joint Structural Biology Group for access and support at the ESRF beamline ID14-4. We thank C. Zubieta, L. Norling and N. Kaldenhoven for their assistance in the early part of this work and R. Read for his efforts with Phaser. We also thank B. Overduin and E. Birney from the EBI for their time and effort in compiling SNP data. Finally, we thank M. Little and M. Piper (University of Queensland, Brisbane, Australia) for the kind gift of full-length Slit2 cDNA. Partial funding for this project was provided by the European Commission Framework 5 Integrated Project 'SPINE' and Framework 6 Integrated Project 'SPINE2Complexes'.

\section{References}

Aricescu, A. R., Lu, W. \& Jones, E. Y. (2006). Acta Cryst. D62, $1243-$ 1250.

Aruga, J., Yokota, N. \& Mikoshiba, K. (2003). Gene, 315, 87-94.

Barash, S., Wang, W. \& Shi, Y. (2002). Biochem. Biophys. Res. Commun. 294, 835-842.

Blom, N., Sicheritz-Ponten, T., Gupta, R., Gammeltoft, S. \& Brunak, S. (2004). Proteomics, 4, 1633-1649.

Brose, K., Bland, K. S., Wang, K. H., Arnott, D., Henzel, W., Goodman, C. S., Tessier-Lavigne, M. \& Kidd, T. (1999). Cell, 96, 795-806.

Carmeliet, P. \& Tessier-Lavigne, M. (2005). Nature (London), 436, 193-200.

Chang, V. T., Crispin, M., Aricescu, A. R., Harvey, D. J., Nettleship, J. E., Fennelly, J. A., Yu, C., Boles, K. S., Evans, E. J., Stuart, D. I., Dwek, R. A., Jones, E. Y., Owens, R. J. \& Davis, S. J. (2007). Structure, 15, 267-273.

Dickson, B. J. \& Gilestro, G. F. (2006). Annu. Rev. Cell Dev. Biol. 22, 651-675.

Durocher, Y., Perret, S. \& Kamen, A. (2002). Nucleic Acids Res. 30, E9.

Emsley, P. \& Cowtan, K. (2004). Acta Cryst. D60, 2126-2132.

Garbe, D. S. \& Bashaw, G. J. (2004). Crit. Rev. Biochem. Mol. Biol. 39, 319-341.

Gouet, P., Courcelle, E., Stuart, D. I. \& Metoz, F. (1999). Bioinformatics, 15, 305-308.

He, X. L., Bazan, J. F., McDermott, G., Park, J. B., Wang, K., TessierLavigne, M., He, Z. \& Garcia, K. C. (2003). Neuron, 38, 177-185.

Howitt, J. A., Clout, N. J. \& Hohenester, E. (2004). EMBO J. 23, 4406-4412.

Huizinga, E. G., Tsuji, S., Romijn, R. A., Schiphorst, M. E., de Groot, P. G., Sixma, J. J. \& Gros, P. (2002). Science, 297, 1176-1179.

Huminiecki, L., Gorn, M., Suchting, S., Poulsom, R. \& Bicknell, R. (2002). Genomics, 79, 547-552.

Hussain, S. A., Piper, M., Fukuhara, N., Strochlic, L., Cho, G., Howitt, J. A., Ahmed, Y., Powell, A. K., Turnbull, J. E., Holt, C. E. \& Hohenester, E. (2006). J. Biol. Chem. 281, 39693-39698.

Ito, H. et al. (2006). Clin. Cancer Res. 12, 3257-3264.

Itoh, A., Miyabayashi, T., Ohno, M. \& Sakano, S. (1998). Brain Res. Mol. Brain Res. 62, 175-186.

Kabsch, W. (1993). J. Appl. Cryst. 26, 795-800.

Kobe, B. \& Kajava, A. V. (2001). Curr. Opin. Struct. Biol. 11, 725-732.

Landau, M., Mayrose, I., Rosenberg, Y., Glaser, F., Martz, E., Pupko, T. \& Ben-Tal, N. (2005). Nucleic Acids Res. 33, W299-W302.

Laskowski, R. A., MacArthur, M. W., Moss, D. S. \& Thornton, J. M. (1993). J. Appl. Cryst. 26, 283-291.

Leahy, D. J., Dann, C. E. III, Longo, P., Perman, B. \& Ramyar, K. X. (2000). Protein Expr. Purif. 20, 500-506.

Letunic, I., Copley, R. R., Pils, B., Pinkert, S., Schultz, J. \& Bork, P. (2006). Nucleic Acids Res. 34, D257-D260.

Little, M., Rumballe, B., Georgas, K., Yamada, T. \& Teasdale, R. D. (2002). Int. J. Dev. Biol. 46, 385-391.

McCoy, A. J., Grosse-Kunstleve, R. W., Storoni, L. C. \& Read, R. J. (2005). Acta Cryst. D61, 458-464. 


\section{research papers}

Morlot, C., Hemrika, W., Romijn, R. A., Gros, P., Cusack, S. \& McCarthy, A. (2007). Acta Cryst. F63, 689-691.

Murshudov, G. N., Vagin, A. A. \& Dodson, E. J. (1997). Acta Cryst. D53, 240-255.

Nguyen Ba-Charvet, K. T., Brose, K., Ma, L., Wang, K. H., Marillat, V., Sotelo, C., Tessier-Lavigne, M. \& Chedotal, A. (2001). J. Neurosci. 21, 4281-4289.

Piper, M., Nurcombe, V., Wilkinson, L. \& Little, M. (2002). Int. J. Dev. Biol. 46, 545-550.
Prasad, A., Fernandis, A. Z., Rao, Y. \& Ganju, R. K. (2004). J. Biol. Chem. 279, 9115-9124.

Rothberg, J. M., Jacobs, J. R., Goodman, C. S. \& Artavanis-Tsakonas, S. (1990). Genes Dev. 4, 2169-2187.

Stein, E. \& Tessier-Lavigne, M. (2001). Science, 291, 1928-1938.

Tessier-Lavigne, M. \& Goodman, C. S. (1996). Science, 274, $1123-$ 1133.

Thompson, J. D., Higgins, D. G. \& Gibson, T. J. (1994). Nucleic Acids Res. 22, 4673-4680. 